\title{
Best Practice for Prevention of Vaccination Common Problems With Antipyretic/Analgesic Medications
}

\author{
Lacey M. Eden \\ Brigham Young University - Provo \\ Meridith G. Lind \\ Brigham Young University - Provo \\ Karlen E. Luthy \\ Brigham Young University - Provo \\ Janelle L. B. Macintosh \\ Brigham Young University - Provo, janelle-macintosh@byu.edu \\ Follow this and additional works at: https://scholarsarchive.byu.edu/facpub \\ Part of the Other Nursing Commons
}

\section{Original Publication Citation}

Eden, L. M., Lind, M. G.**, Luthy, K. E., \& Macintosh, J. L. (2017). Best practice for prevention of vaccination common problems with antipyretic/analgesic medications. The Journal for Nurse Practitioners, 13(7), 462-467.

\section{BYU ScholarsArchive Citation}

Eden, Lacey M.; Lind, Meridith G.; Luthy, Karlen E.; and Macintosh, Janelle L. B., "Best Practice for Prevention of Vaccination Common Problems With Antipyretic/Analgesic Medications" (2017). Faculty Publications. 5204.

https://scholarsarchive.byu.edu/facpub/5204

This Peer-Reviewed Article is brought to you for free and open access by BYU ScholarsArchive. It has been accepted for inclusion in Faculty Publications by an authorized administrator of BYU ScholarsArchive. For more information, please contact ellen_amatangelo@byu.edu. 


\title{
Best Practice for Prevention of
}

\section{Vaccination Common Problems With} Antipyretic/Analgesic Medications Lacey M. Eden, MS, NP-C, Meridith G. Lind, MS, FNP, Karlen E. Luthy, DNP, FNP, and Janelle L.B. Macintosh, PhD, RN

\begin{abstract}
Nurse practitioners are on the front lines providing parental education regarding vaccines. Some reasons for vaccine hesitancy include the potential common mild problems of vaccine administration, such as pain and/or elevated temperature. According to research, prophylactic administration of an antipyretic/analgesic medication, such as acetaminophen, reduces common mild problems related to vaccines when administered before or at the time of vaccination. Additionally, the evidence that prophylactic administration of antipyretic/analgesic medication decreases antibody response to vaccinations is insufficient at this time. However, this research has led to some confusion on best practice guidelines. This review of the literature resulted in recommendations that nurse practitioners should reassure parents that an antipyretic/analgesic medication will not reduce the immune response and can be administered to prevent or reduce common mild problems of vaccinations, which may then reduce vaccine hesitancy among parents.
\end{abstract}

Keywords: antipyretic, analgesic, immunization, prophylactic, vaccine (C) 2017 Elsevier Inc. All rights reserved.

T n 2014, 91\% of American children under age 6 received a well-child examination. ${ }^{1}$ The

Advisory Committee on Immunization

Practices $^{2}$ and the American Academy of Pediatrics ${ }^{3}$ recommend the administration of vaccinations during most routine well-child examinations. According to the Centers for Disease Control and Prevention, the majority of childhood vaccinations are administered during the first 18 months of life. ${ }^{4}$ However, sometimes infants and young children experience common mild problems from vaccines such as elevated temperature, tenderness, and/or swelling at the injection site.

Parents play an integral part in their child's health care; thus, it is vital that nurse practitioners (NPs) use

American Association of Nurse Practitioners (AANP) members may receive 1.0 continuing education contact hours, including 1.0 pharmacology credit, approved by AANP, by reading this article and completing the online posttest and evaluation at aanp.inreachce.com. motivational interviewing to understand parental concerns regarding vaccine administration. ${ }^{6}$ Although there are many reasons for parental vaccine hesitancy, mild common problems related to vaccines are often sighted as a reason to exempt or delay vaccine administration. ${ }^{5,7}$ If sighted as a concern, NPs oftentimes recommend the administration of an antipyretic/analgesic medication to infants and young children before or at the time of routine vaccinations. ${ }^{8}$ However, this practice is sometimes surrounded by controversy. ${ }^{5}$ Although it is wellknown that antipyretic/analgesic medications, such as acetaminophen, can be safely administered for mild common problems related to vaccinations, 1 study suggests this practice may negatively affect the child's ability to produce an effective immune response to vaccines.' Conversely, other studies find antipyretic/ analgesic medications effectively treat mild common problems associated with vaccines, such as elevated 
temperature and discomfort, and do not negatively affect the immune response. ${ }^{10}$

As health care providers, NPs should provide evidence-based education to parents regarding the potential benefits and risks of antipyretic/analgesic medication and vaccination. However, there are few studies that have investigated the immune response and antipyretic/analgesic medication administration, and the findings in these studies are inconsistent. ${ }^{9-11}$ The research regarding the use of antipyretic/ analgesic medications to treat mild common problems related to vaccines is outdated. Therefore, the purposes of this article are to 1) review the literature regarding the prophylactic use of antipyretic/analgesic medications before or at the time of vaccination administration, 2) make a clinical recommendation for the NP regarding best practice, and 3) call for more research to resolve the question regarding antibody response and antipyretic/analgesic treatment for mild common problems associated with vaccines.

\section{METHODS}

CINAHL, MEDLINE, and the Cochrane Library were searched to identify articles examining prophylactic antipyretic/analgesic medication use for vaccination administration. Additionally, select websites were reviewed, including the Centers for Disease Control and Prevention and the US Department of Health and Human Services websites. Search terms included acetaminophen, ibuprofen, paracetamol, antipyretic, prophylactic, immunization, vaccine, fever, febrile, adverse side effects or reactions, pain, swelling, irritability, fussiness, and antibody. Only randomized controlled trials (RCTs) published in the English language were included for review. Articles were included if published since 1987, which was the year of the first published RCT on this topic. Because the focus of this literature review was on antipyretic/analgesic medication use in infants and children before, at the time of, or after vaccination administration, articles regarding antipyretic/analgesic medication use for adolescent and adult vaccinations were excluded.

\section{RESULTS}

Thirteen articles met the inclusion criteria. Although all 13 studies included research regarding the effect of antipyretic/analgesic medications on vaccine-related mild common problems, some researchers evaluated the effect of antipyretic/analgesic medications on the patient's ability to produce a healthy antibody response after vaccination.

The type of antipyretic/analgesic medication differed from study to study. Although acetaminophen and ibuprofen are available in the United States, ibuprofen is only approved for use in children 6 months and older. This is significant because many vaccines are given before 6 months of age. Additionally, paracetamol, although not available in the US, was 1 of the antipyretic/analgesics used in some of the studies. Furthermore, the type of vaccine administered and the number of vaccines in the series varied from study to study.

Three main outcomes were measured in the 13 studies, namely, the effect of antipyretic/analgesic medications on local vaccine reactions, systemic vaccine reactions, and antibody response. Nine studies focused on the antipyretic/analgesic medication effect on local vaccine reactions, specifically vaccine site redness, swelling, and pain. ${ }^{9,12-19}$ Eleven studies evaluated the effect of antipyretic/analgesic medications on systemic vaccine reactions, such as elevated temperature, fussiness, and sleep duration. 9,10,12-15,17-21 Researchers also measured the effect of antipyretic/analgesic medications on antibody response in 3 studies. ${ }^{9-11}$

\section{Local Vaccine Reactions}

Redness and Swelling. Eight RCTs addressed the relationship between redness and swelling at the vaccination site and antipyretic/analgesic medication administration..$^{9,13-19}$ In 6 RCTs, researchers found a decrease in redness and swelling at the vaccination site when antipyretic/analgesic medication was administered before or at the time of vaccination although the findings were not always statistically significant. ${ }^{9,13-15,17,18}$ Rose et $\mathrm{al}^{18}$ found the reduction of redness and swelling with prophylactic antipyretic/ analgesic medication varied depending on the type of vaccine being administered and the number of doses in the vaccine series. A reduction of redness and swelling was observed in all doses of the series for both the pneumococcal conjugate vaccine, 7-valent (PCV-7), and the combination vaccine, diphtheria, tetanus, 
acellular pertussis-inactivated poliovirus/haemophilus influenza type b vaccine (DTPa-IPV/Hib). However, the statistical significance of decreased redness and swelling varied from $P=.475$ with the first dose of PCV-7 to $P=.017$ with the second dose of PCV-7. Similarly, the statistical significance of decreased redness and swelling varied from $P>.99$ with the first dose of DTPa-IPV/Hib to $P=.115$ with the second dose. Similarly, 4 studies found $1 \%$ to $13 \%$ less reports of swelling in the treatment group compared with the control group. ${ }^{9}$

Findings were only statistically significant in 1 study in which Ipp et al ${ }^{15}$ noted a $9 \%$ reduction $(P<.025)$ in cases of vaccine-related redness at the injection site in patients who received acetaminophen before or at the time of vaccination when compared with the placebo group. Ipp et al also found a decreased incidence of swelling and that patients receiving prophylactic acetaminophen were less likely to restrict movement of their legs; however, these findings were not statistically significant. Contrastingly, 2 studies found no consistent difference in redness or swelling between the group receiving the antipyretic/analgesic and the placebo group. ${ }^{16,19}$

Pain. The effect of analgesic medication on vaccine-related pain at the injection site was evaluated in 6 studies. ${ }^{9,13-15,17,19}$ There was a statistically significant decrease in vaccine-related pain in patients who received prophylactic treatment with an analgesic medication in 2 of the studies. In a series of 3 vaccine administrations, Díez-Domingo et $\mathrm{a}^{13}$ found that mild pain was less frequent and less intense in the group receiving prophylactic antipyretic/analgesic compared with the control group after the first 2 vaccination doses $(P<.05) .{ }^{13}$ Additionally, Ipp et $\mathrm{al}^{15}$ found that parents reported vaccine-related pain less frequently in children who received an antipyretic/analgesic at the time of vaccination $(P<.001)$. Four studies with sample sizes from $\mathrm{N}=270$ to $\mathrm{N}=459^{9,14,17,19}$ found no statistically significant decreases in vaccine-related pain at the injection site with prophylactic antipyretic/ analgesic administration.

\section{Systemic Vaccine Reactions}

Elevated Temperature. Although 10 studies investigated the effect of antipyretic/analgesic medication on elevated temperature secondary to vaccination, $9,10,12-15,17-19,21$ the timing of medication administration varied among the studies.

Administration Scheduled. Eight studies investigated the effects of antipyretic/analgesic medication administration before, at the time of, or after vaccination. Four of the 8 studies found a significant reduction in elevated temperature. ${ }^{14,15,17,18}$ Hayat et $\mathrm{al}^{14}$ studied the effect of prophylactic antipyretic/ analgesic medication on infants aged 6 weeks, 10 weeks, and 14 weeks as well as toddlers aged 18 months. When an antipyretic/analgesic medication was given 1 hour before vaccination and again 6,12, and 18 hours after vaccination, the incidence of elevated temperature was significantly decreased in every age group $(P<.02)$. Similarly, Lewis et $\mathrm{al}^{17}$ reported that elevated temperatures were less frequent in patients who received an antipyretic/analgesic medication before vaccination and again at 3, 7, 12, and 18 hours after vaccination. Although not statistically significant, participants who were 2 months old showed the most marked difference, with nearly a $60 \%$ reduction in elevated temperature $(P=.065)$ compared with $50 \%$ in the control group. ${ }^{12}$

In another study, there was a statistically significant reduction in vaccine-related elevated temperatures $(P<.001)$ when the antipyretic/analgesic medication was administered at the same time as the vaccination. ${ }^{18}$ When antipyretic/analgesic medication was given immediately after vaccination, Ipp et $\mathrm{al}^{15}$ found a significant reduction $(P<.0005)$ in elevated temperatures in infants aged 2 to 6 months. Contrastingly, in 4 other studies with sample sizes from $\mathrm{N}=263$ to $\mathrm{N}=374,{ }^{10,13,19,21}$ researchers did not find a statistically significant difference with the incidence of elevated temperature between patients who received antipyretic/analgesic medication before or at the time of vaccination and patients who were not treated with antipyretic/analgesic medication.

Administration as needed. When parents could administer an antipyretic/analgesic medication on an as-needed basis, rather than prophylactically, there was a statistically significant higher incidence of elevated temperature $(P=.04)$ during the first 6 hours after vaccination. ${ }^{12}$ 
Administration in series. Prymula et $\mathrm{al}^{11}$ measured elevated temperatures in infants after receiving the initial vaccination for DTPa-IPV/Hib and again after receiving the second vaccination in each of the series. An elevated temperature $>39.5^{\circ} \mathrm{C}$ was uncommon $(\leq 2 \%)$ in both the placebo and antipyretic/analgesic medication groups for both the initial and second vaccination. However, an elevated temperature $>38^{\circ} \mathrm{C}$ was $24 \%$ higher in the placebo group after the initial vaccine and $22 \%$ higher after the second vaccine in the series.

Fussiness. The effect of prophylactic antipyretic/analgesic medication on behavioral changes postvaccination, such as fussiness or irritability, was researched in 8 studies. ${ }^{9,12-15,17,19,21}$ Fewer incidents of postvaccination fussiness were reported in 4 studies with the use of prophylactic antipyretic/analgesic medication. ${ }^{14,15,17,21}$ In 3 of these studies, researchers reported the reduction in fussiness was statistically significant $\left(P<.0001,{ }^{15} P=.01,{ }^{17}\right.$ and $\left.P<.02^{14}\right)$. Jackson et $\mathrm{al}^{21}$ reported a $10 \%$ reduction in the incidence of postvaccination fussiness with concurrent administration of an antipyretic/analgesic medication. Although the findings of Jackson et $\mathrm{al}^{21}$ were not statistically significant, a $10 \%$ reduction in fussiness could be considered clinically significant.

Díez-Domingo et $\mathrm{al}^{13}$ measured postvaccination fussiness with the administration of an antipyretic/ analgesic medication for 3 separate diphtheria, tetanus, pertussis (DTP) vaccinations in a series. Interestingly, there was a statistically significant decrease in postvaccination fussiness $(P<.05)$, but only for the first DTP vaccination in the series. The second and third DTP vaccinations in the series had no statistically significant difference in fussiness between the children who received an antipyretic/ analgesic medication before vaccination and those who did not. ${ }^{13}$

In contrast, researchers reported no statistically significant change in fussiness between children who received prophylactic treatment with an antipyretic/ analgesic and those who received no treatment. However, there were $4.7 \%$ to $8.8 \%$ fewer episodes of fussiness in the groups who received prophylactic treatment with an antipyretic/analgesic medication before vaccination when compared with the nontreatment groups. ${ }^{9,12,19}$ Nevertheless, the differences between groups were not statistically significant.

Sleep. Interference with routine sleep patterns postvaccination was evaluated in only 1 study. ${ }^{20}$ Franck et $\mathrm{al}^{20}$ reported that infants who received prophylactic treatment with an antipyretic/analgesic medication slept less after vaccination when compared with infants who were not treated with an antipyretic/analgesic medication prophylactically. The researchers speculated that although higher temperature may be a primary mechanism for increased sleep, prophylactic treatment with antipyretic/analgesic decreases fever, thus decreasing sleep time. Interestingly, and statistically significant, was the timing of the vaccine administration. Infants receiving vaccinations after 1:30 PM had a longer sleep duration regardless of whether or not they received the antipyretic/analgesic medication $(P<.007) .{ }^{20}$

\section{Antibody Response}

Researchers studied prophylactic treatment with antipyretic/analgesic medications before or at the time of vaccination and its effect on the ability to mount an appropriate postvaccination immune response. ${ }^{9-11}$ Uhari et $\mathrm{al}^{10}$ reported no statistically significant difference in immunoglobulin $G$ antibodies in 5-month-old infants who received a prophylactic dose of antipyretic/analgesic medication (acetaminophen) when evaluated 6 weeks after receiving the DTP vaccine. However, in a sentinel study, Prymula et $\mathrm{al}^{9}$ found statistically significant lower antibody geometric mean concentrations in patients who received an antipyretic/analgesic medication (paracetamol) before vaccination. The vaccine antibody response remained blunted with the administration of the next vaccine in the series in children who received a dose of antipyretic/ analgesic medication. ${ }^{9}$ However, during a follow-up study, ${ }^{11}$ researchers found the effect of antipyretic/ analgesic (paracetamol) administration before or at the time of vaccination had a questionable effect on antibody response. Additionally, the lower immune response did not persist and was described as "transient in nature." Consequently, Prymula et $\mathrm{al}^{11}$ concluded that decreases in antibody response 
after antipyretic/analgesic medication did not always occur or persist.

\section{DISCUSSION}

Short-term vaccination side effects can be concerning for both parents and providers. ${ }^{22}$ Despite unpleasant, albeit non-life-threatening, localized and systemic vaccine-related mild common problems, the US Department of Health and Human Services ${ }^{23}$ maintains that vaccines are safe and effective, save lives, protect others through herd immunity, save time and money, and help protect future generations from potentially deadly vaccine-preventable diseases. Nevertheless, if parents are anxious regarding vaccine-related mild common problems, namely, pain, they may be reluctant to have their children vaccinated according to the current recommended schedule. ${ }^{4,24}$ Therefore, it is important to educate parents about how to effectively treat common vaccine-related mild common problems.

The potential for postvaccination fever is common and worrisome to some parents, although some parents fear the potential for seizure secondary to fever rather than the fever itself. ${ }^{25}$ Interestingly, although there is a slightly increased risk of febrile seizure with the measles, mumps, and rubella and measles, mumps, rubella, and varicella vaccines, ${ }^{26}$ the American Academy of Pediatrics ${ }^{27}$ recommends prophylactic treatment with an antipyretic/analgesic medication only for the diphtheria, tetanus, acellular pertussis vaccination when there is a personal or family history of febrile seizures. Because observing a febrile seizure can be disturbing, parents should be educated about the potential for febrile seizures with vaccination and simultaneously reassured that febrile seizures are generally shorter than 1 to 2 minutes, do not cause any permanent harm, and are uncommon after vaccinations. ${ }^{26}$ In addition, education should include the fact that administering an antipyretic/ analgesic medication does not actually reduce the chance of the child having a febrile seizure. ${ }^{28}$ Thus, in the event the child does have a febrile seizure after vaccine administration, the child should be seen by his or her health care provider.

Currently, evidence is insufficient to suggest an altered immune response with the administration of a prophylactic antipyretic/analgesic with vaccinations.
The controversy of holding the administration of an antipyretic/analgesic medication because of the potential to interfere with immunity is based on 1 , isolated study ${ }^{9}$ investigating only paracetamol as the antipyretic/analgesic. However, it should be noted the single study suggesting an adverse effect on antibody response still produced therapeutic levels in the majority of the participants. ${ }^{9}$ This finding suggests that the antibody response, although potentially decreased, would not necessarily be impaired enough to be harmful to patients. Furthermore, the clinical significance of this single study is unknown ${ }^{29}$ as well as the effect on immunity when a complete series of vaccinations is administered. Thus, further exploration of a relationship between an antipyretic/ analgesic medication and a decreased antibody response is needed.

\section{IMPLICATIONS FOR NPS}

Although it is well-known that administering prophylactic antipyretic/analgesic medication can effectively reduce vaccine-related mild common problems, there are still questions regarding the antibody response. Currently, there is not enough evidence to verify that prophylactic antipyretic/analgesic medication decreases the antibody immune response. Thus, it is imperative for NPs to accurately understand the literature and properly educate families about vaccinations while prioritizing parental concerns.

Specifically, when parents sight vaccine-related mild common problems as a barrier to vaccination, NPs should discuss antipyretic/analgesic medication use before or at the time of vaccination. Knowing their children might be able to avoid discomfort may ease parental anxiety and encourage them to adhere to the recommended vaccination schedule. However, not all children develop vaccine-related mild common problems. Thus, it may not be necessary to administer an antipyretic/analgesic agent prophylactically. Delaying the administration of antipyretic/analgesic medication until the child exhibits vaccine-related mild common problems would be appropriate.

\section{CONCLUSION}

Educating parents about the use of prophylactic antipyretic/analgesic medication at the time of vaccination is an essential role of the NP. Currently 
there is little research regarding antipyretic/analgesic administration and the antibody response; furthermore, the evidence is not consistent. Thus, it is imperative to call for more research on this topic to better implement best practices with vaccine administration.

However, with the available evidence, the recommended best practice is for NPs to teach parents to administer an antipyretic/analgesic medication prophylactically if concerned about vaccine-related mild common problems or after the mild common problems have manifested. Parents can be reassured that there is not yet enough evidence to avoid an antipyretic/analgesic based on its interference with the antibody response. Nevertheless, NPs should remain up-to-date with the literature so they can wisely counsel patients and families regarding vaccine-related mild common problems and the administration of antipyretic/analgesic medication. JNP

\section{References}

1. Well-child visits. Child Trends Databank. https://www.childtrends.org/ indicators/well-child-visits/. October 2014. Accessed March 20, 2016.

2. Advisory committee on immunization practices recommended immunization schedules for persons aged 0 through 18 years-United States 2016. Morbidity and Mortality Weekly Report. https://www.cdc.gov/mmwr/volumes/ 65/wr/mm6504a4.htm. February 2016. Accessed April 1, 2017.

3. Recommendations for preventive pediatric health care. American Association of Pediatrics website. https://www.aap.org/en-us/professional-resources/ practice-support/Pages/PeriodicitySchedule.aspx. January 2016. Accessed October 3, 2016

4. Possible side effects from vaccines. Centers for Disease Control and Prevention. Website. http://www.cdc.gov/vaccines/vac-gen/side-effects.html. December 2, 2016. Accessed January 6, 2017

5. Pedulla MN. Prophylactic use of antipyretic agents with childhood immunizations and antibody response: Reason for concern? J Pediatr Health Care. 2012;26(3):200-203.

6. Leask J, Kinnersley P, Jackson C, Cheater F, Bedford H, Rowles G. Communicating with parents about vaccination: a framework for health professionals. BMC Pediatr. 2012;12:154.

7. Anderson VL. Promoting childhood immunizations. J Nurse Pract. 2015;11:1-10

8. Homme JH, Fischer PR. Prophylactic paracetamol at the time of infant vaccination reduces the risk of fever but also reduces antibody response. Evid Based Med. 2010;15:50-51.

9. Prymula R, Siegrist CA, Chlibek R, et al. Effect of prophylactic paracetamol administration at time of vaccination on febrile reactions and antibody responses in children: two open-label, randomised controlled trials. Lancet. 2009;374:1339-1350.

10. Uhari M, Hietala J, Vilijanen M. Effect of prophylactic acetaminophen administration on reaction to DTP vaccination. Acta Paediatr Scand. 1988;77:747-751.

11. Prymula R, Habib A, François N. Immunological memory and nasopharyngeal carriage in 4-year-old children previously primed and boosted with 10-valent pneumococcal non-typeable haemophilus influenzae protein $\mathrm{D}$ conjugate vaccine (PHiD-CV) with or without concomitant prophylactic paracetamol. Vaccine. 2013;31(16):2080-2088.

12. Dhingra B, Mishra D. Immediate versus as-needed acetaminophen for postimmunisation pyrexia. Ann Trop Paediatr. 2011;31(4):339-344.

13. Díez-Domingo J, Planelles MV, Baldó JM, et al. Ibuprofen prophylaxis for adverse reactions to diphtheria-tetanus-pertussis vaccination: a randomized trial. Curr Ther Res. 1998;59(8):579-588.
14. Hayat H, Khan PS, Hayat G. The effect of prophylactic paracetamol administration on adverse reactions following DTP vaccination. East $J$ Med. 2011;16:258-260.

15. Ipp MM, Gold R, Greenberg S, et al. Acetaminophen prophylaxis of adverse reactions following vaccination of infants with diphtheriapertussis-tetanus toxoids-polio vaccine. Pediatr Infect Dis J. 1987;6(8): $721-725$.

16. Jackson LA, Dunstan M, Starkovich P. Prophylaxis with acetaminophen or ibuprofen for prevention of local reactions to the fifth diphtheria-tetanus toxoids-acellular pertussis vaccination: a randomized, controlled trial. Pediatrics. 2006;117(3):620-625.

17. Lewis K, Cherry JD, Sachs MH, et al. The effect of prophylactic acetaminophen administration on reactions to DTP vaccination. Am J Dis Child. 1988;142(1):62-65.

18. Rose MA, Juergens C, Schmoele-Thoma B. An open-label randomized clinical trial of prophylactic paracetamol coadministered with 7-valent pneumococcal conjugate vaccine and hexavalent diphtheria toxoid, tetanus toxoid, 3component acellular pertussis, hepatitis $B$, inactivated poliovirus, and Haemophilus influenzae type b vaccine. BMC Pediatr. 2013;13:1.

19. Yalçin SS, Gümüş A, Yurdakök K. Prophylactic use of acetaminophen in children vaccinated with diphtheria-tetanus-pertussis. World J Pediatr. 2008;4:127-129.

20. Franck L, Gay $C L$, Lynch $M$, Lee $K A$. Infant sleep after immunization: randomized controlled trial of prophylactic acetaminophen. Pediatrics. 2011;128:1100-1108.

21. Jackson LA, Peterson D, Dunn J, et al. A randomized placebo-controlled trial of acetaminophen for prevention of post-vaccination fever in infants. PLoS One. 2011;6(6):1-7.

22. Paul SP, Whibley J. Paracetamol prophylaxis: what the evidence says. Pract Nurs. 2010;21(10):530-531.

23. Five important reasons to vaccinate your child. U.S. Department of Health and Human Services website. https://www.vaccines.gov/more_info/features/ five-important-reasons-to-vaccinate-your-child.html 2015. February 16, 2016. Accessed October 24, 2016

24. Luthy KE, Beckstrand RL, Peterson NE. Parental hesitation as a factor in delayed childhood immunization. J Pediatr Health Care. 2009;23(6): 388-393.

25. Martins M, Abecasis F. Healthcare professionals approach paediatric fever in significantly different ways and fever phobia is not just limited to parents. Acta Paediatr. 2016;105(7):829-833.

26. Childhood vaccines and febrile seizures. Centers for Disease Control and Prevention website. http://www.cdc.gov/vaccinesafety/concerns/febrile-seizures .html. August 28, 2015. Accessed September 27, 2016

27. Pickering LK, Baker CJ, Kimberlin DW. Red Book: 2012 Report of the Committee on Infectious Disease. 29th ed. Elk Grove Village, IL: American Academy of Pediatrics; 2012. https://redbook.solutions.aap.org/DocumentLibrary/RB12 interior.pdf. Accessed September 302016.

28. Treating febrile seizures. National Health Service Choices website. http:/ www.nhs.uk/Conditions/Febrileconvulsions/Pages/Treatment.aspx. January 2014. Accessed September 23, 2016

29. Drutz TK. Standard immunizations for children and adolescents. UpToDate website. http://www.uptodate.com. Accessed October 3, 2016.

All authors are affiliated with SWKT College of Nursing at Brigham Young University in Provo, UT. Lacey M. Eden, MS, $\mathrm{NP}-\mathrm{C}$, is an assistant teaching professor and can be reached at Lacey_eden@byu.edu. Meridith G. Lind, MS, FNP, is a student. Karlen E. Luthy, DNP, FNP, is an associate professor. Janelle L.B. Macintosh, PhD, RN, is an assistant professor. In compliance with national ethical guidelines, the authors report no relationships with business or industry that would pose a conflict.

\section{$1555-4155 / 17 / \$$ see front matter}

(c) 2017 Elsevier Inc. All rights reserved.

http://dx.doi.org/10.1016/j.nurpra.2017.05.005 